\title{
Avaliação da destreza dos dedos e da força de preensão máxima em crianças com dislexia desenvolvimental
}

\author{
Paulo Barbosa de FREITAS* \\ Jose Angelo BARELA*/** \\ Sabrina Tiago PEDÃO* \\ Kauê Almeida LIMA* \\ Cristina Lopes RIBEIRO*
}

*Instituto de Ciências

da Atividade Física e

Esporte, Universidade Cruzeiro do Sul,

São Paulo, SP, Brasil

**Departamento de

Educação Física,

Universidade Estadual

Paulista Júlio de

Mesquita Neto

São Paulo, SP, Brasil.

\section{Resumo}

A dislexia caracteriza-se pela dificuldade de aprendizagem da leitura, escrita e soletração, sem uma causa aparente específica. Crianças e adultos com dislexia apresentam também déficits em diferentes tarefas sensório-motoras. Porém, não existe consenso quanto o efeito da dislexia na destreza dos dedos e se há alterações puramente motoras em individuos acometidos por essa desordem. 0 objetivo do estudo foi comparar crianças com e sem dislexia quanto à destreza dos dedos e à capacidade de geração de força máxima. Trinta crianças com dislexia e 30 sem dislexia, entre 8 e 14 anos, realizaram o teste dos nove pinos nos buracos (9-PnB) para avaliação da destreza manual e o teste força de preensão palmar máxima, ambos com a mão dominante. Elas foram instruídas a realizar o teste dos 9- $\mathrm{PnB}$ o mais rápido possível e em seguida produzir força de preensão máxima $\left(\mathrm{FP}_{\mathrm{Max}}\right)$ no dinamômetro hidráulico Jamar ${ }^{\circledR}$. 0 menor tempo e a maior $\mathrm{FP}_{\mathrm{Max}}$ registradas em três tentativas foram utilizadas para as análises estatísticas. Os resultados revelaram que as crianças com dislexia são mais lentas na execução do teste dos 9-PnB, porém apresentam similar capacidade de geração de $\mathrm{FP}_{\mathrm{Max}}$ que crianças não disléxicas. Esses resultados indicam que as diferenças no desempenho em testes motores observadas entre crianças com dislexia e sem dislexia não têm origem no sistema motor e sim no modo com que a criança com dislexia processa as informações sensoriais e as transforma em respostas motoras para produzir ações.

Palavras-Chave: Função Manual; Coordenação; Controle Motor; Habilidade Manipulativa.

\section{Introdução}

Dislexia desenvolvimental é caracterizada pela dificuldade de aprendizagem da leitura, escrita e soletração, sem uma causa aparente específica e que não está associada a déficits de inteligência, falta de instrução ou oportunidade educacional e outro problema médico aparente ${ }^{1-3}$. A dislexia afeta uma parcela considerável da população, sendo que o número de crianças com dislexia pode ser superior a $10 \%{ }^{4,5}$. Este problema traz consequências negativas na atuação e no desempenho escolar dessas crianças, levando, em casos extremos, a evasão escolar.

A dislexia é uma desordem neurocomportamental e de origem genética ${ }^{2,3,6}$. Porém, não há consenso sobre o local (ou locais) de origem dessa desordem.
Para um grupo de pesquisadores, os problemas enfrentados por disléxicos estariam relacionados a disfunçóes nos centros nervosos responsáveis pela linguagem ${ }^{5,7,8}$, o que afetaria o modo como o sistema nervoso processa as caracteristicas fonológicas das palavras quando as mesmas são faladas. Para outros pesquisadores, a dislexia desenvolvimental seria causada por déficits visuais, especificamente por alterações na formação das células $\mathrm{M}$ ou magnocélulas que formam o caminho retinocortical $^{9-13}$. Finalmente, para outro grupo, a dislexia seria causada por alteraçóes estruturais e funcionais do cerebelo, responsável por integrar os estímulos sensoriais provenientes de diferentes 
canais e utilizar as informaçóes provenientes desses estímulos para a produção de ações motoras ${ }^{13-18}$.

Essas diferentes posiçóes quanto a causa da dislexia surgiram após a observação de que as crianças disléxicas náo apresentam apenas problemas fonológicos ligados à leitura, soletração e escrita. Vários estudos têm mostrado que crianças e adultos com dislexia apresentam também déficits em diferentes tarefas ${ }^{1,13,14,19}$ e a causa seria mudanças estruturais e funcionais do cerebelo e também nas vias magnocelulares do sistema visual ${ }^{11,20,21}$ e que estes problemas motores persistem na adolescência ${ }^{1}$ e na vida adulta ${ }^{22}$. Especificamente, crianças com dislexia têm desempenhos inferiores em testes de destreza dos dedos que envolvem a colocaçáo de pinos em buracos. Leslie, Davidson e Batey ${ }^{19}$ observaram que crianças com dislexia, com idade entre 9 e 13 anos, têm desempenho inferior (menor número de pinos transportados em $30 \mathrm{~s}$ ) ao das crianças sem dislexia e com a mesma idade no teste Perdue Peg Board tanto com a mão dominante quanto com a não dominante. Ainda, FAWCETT e NICOLSON ${ }^{1}$ mostraram diferença entre disléxicos e não-disléxicos pareados pela idade em um teste de pinos. Porém, Getchell et al. ${ }^{23}$ não observaram diferenças entre crianças disléxicas e náo-dislexicas na parte de destreza manual do teste MABC (Movement Assessment Battery for Children). Considerando esses resultados conflitantes, há necessidade de melhor avaliar o desempenho de crianças com dislexia nos testes dos pinos que são utilizados para avaliação da destreza dos dedos e coordenaçáo sensório-

\section{Método}

\section{Participantes}

Participaram do estudo trinta crianças com dislexia ( 20 meninos e 10 meninas) entre 8 e 14 anos e trinta crianças sem problemas de leitura e escrita, todas pareadas por idade, sexo e preferência manual. As crianças tinham em média ( \pm desvio padráo) $10,89( \pm 1,42)$ anos de idade, 44,39 $( \pm 15,06) \mathrm{kg}$ de massa corporal e $1,47( \pm 0,11)$ metros de estatura, enquanto que as crianças do grupo controle tinham 10,92 $( \pm 1,56)$ anos de idade, 43,16 $( \pm 17,36) \mathrm{kg}$ de massa corporal e $1,47( \pm 0,12)$ metros de estatura. As crianças com dislexia foram diagnosticadas e encaminhadas pelo Centro de Especialização em Fonoaudiologia Clínica (CEFAC) de São Paulo e pela Associação Brasileira de Dislexia (ABD). As crianças do grupo motora. Assim, um dos objetivos do presente estudo foi comparar a destreza dos dedos de crianças disléxicas e crianças não-disléxicas. A hipótese testada foi que crianças com dislexia desenvolvimental apresentariam pior destreza dos dedos quando comparadas as crianças não-disléxicas.

Além da destreza dos dedos, também foi avaliada a capacidade de crianças com dislexia em gerar força de preensão palmar máxima. A tarefa de realizar força de preensão palmar máxima é uma tarefa predominantemente motora, com mínima influência dos sistemas sensoriais no resultado obtido. Desse modo, investigar o desempenho de crianças com dislexia em uma tarefa predominantemente motora é importante para determinar se essa população também apresenta déficts motores além dos já conhecidos problemas na execução de tarefas sensório-motoras. Até o momento, não encontramos qualquer estudo com crianças disléxicas que tenha avaliado a capacidade de geraçáo de força de preensão palmar máxima. Assim, um objetivo adicional desse estudo foi comparar a capacidade de geraçáo de força de preensão palmar máxima em crianças com e sem dislexia diagnosticada. Nesse caso, a hipótese testada foi que crianças com dislexia não apresentariam diferenças na produção de força máxima das crianças sem dislexia, já que possíveis problemas que essas crianças experienciam estariam diretamente relacionados com o processamento das informações sensoriais e integração dessas informações com o sistema motor $^{13,14,24}$.

controle foram recrutadas de escolas públicas e particulares da regiáo metropolitana de São Paulo. Essas crianças estavam matriculadas em escolas públicas e particulares, tinham a capacidade de leitura compatível com a idade e bom rendimento escolar atestado pelos respectivos professores. Todas as crianças participantes do estudo receberam permissão dos seus pais ou responsáveis por meio da assinatura de um termo de consentimento livre e esclarecido aprovado pelo Comitê de Ética em Pesquisa da Universidade Cruzeiro do Sul.

\section{Procedimentos}

Para determinar a preferência manual de cada criança foi utilizado o Inventário de Dominância Manual de Edimburgo ${ }^{25}$ traduzido para a língua 
portuguesa. Os resultados mostraram que todas as crianças tinham a mão direita como dominante. Em seguida, as crianças foram instruídas a realizar dois testes com a mão dominante, sendo um teste para avaliar a destreza dos dedos e outro para avaliar a força de preensão palmar máxima $\left(\mathrm{FP}_{\mathrm{Max}}\right)$. O teste dos nove pinos nos buracos (9-PnB, em inglês nine hole peg test, Rolyan ${ }^{\oplus}$ ) foi utilizado para avaliar a destreza dos dedos e, indiretamente, a coordenação sensório-motora. O teste dos 9-PnB é realizado em um tabuleiro de plástico azul dividido em duas partes. Em uma das metades há um recipiente côncavo onde são colocados os 9 pinos $(6,4 \mathrm{~mm}$ de diâmetro e $32 \mathrm{~mm}$ de comprimento) e na outra metade existe 9 buracos $(\mathrm{d}=7,1$ e $\mathrm{l}=13 \mathrm{~mm})$, dispostos em 3 colunas por três linhas distantes $3,2 \mathrm{~cm}$ um do outro. No início do teste, os pinos foram colocados nesse recipiente côncavo. As crianças foram instruídas a usar a mão não testada para segurar e dar estabilidade ao tabuleiro de teste e usar a outra mão para pegar os pinos, um a um, e encaixá-los no buraco e retirálos imediatamente após a colocação do $9{ }^{\circ}$ pino, recolocando-os no recipiente de origem, também um por vez ${ }^{26-28}$. Cada criança realizou três tentativas e o tempo (em segundos) gasto para a execução da tarefa foi registrado por um cronômetro digital. O menor tempo realizado pela criança nas três tentativas foi utilizado como variável dependente na análise estatística.

Após realizarem o teste dos 9- $\mathrm{PnB}$, as crianças realizaram o teste de $\mathrm{FP}_{\mathrm{Max}}$, utilizando o dinamômetro hidráulico de mão do tipo Jamar ${ }^{\oplus 26,29}$. Seguindo as recomendações da American Society of Hand Therapists (ASHT) e de estudo normativo ${ }^{29}$, o equipamento foi ajustado individualmente ao tamanho da mão de cada criança. Para a realizaçáo do teste, cada criança foi instruída a sentar-se em uma cadeira de forma que pudesse estar confortável e com seus pés apoiados no chão. O membro superior dominante juntamente com o punho foi posicionado de forma neutra (i.e., braço em adução ao lado do tronco, cotovelo em flexão e antebraço pronado a $90^{\circ}$ ). Foi solicitado para as crianças realizarem o máximo de força possível apertando as hastes do dinamômetro uma contra a outra durante 4 segundos, relaxando posteriormente ao comando do experimentador. Novamente, cada criança realizou três tentativas com um intervalo de 1 minuto entre cada tentativa a fim de evitar que uma possível fadiga muscular pudesse influenciar nos resultados. A variável dependente utilizada para análise dos dados foi o maior valor registrado da $\mathrm{FP}_{\mathrm{Max}}$ (em kgf) nas três tentativas realizadas.

\section{Análise estatística}

Para análise dos dados foi utilizado o programa estatístico SPSS (SPSS for Windows Version 18). Após observarmos que os valores do tempo de execução do teste dos 9-PnB e da $\mathrm{FP}_{\mathrm{Max}}$ tinham distribuição normal (Teste de Shapiro-Wilk) utilizamos dois testes $t$ de student para amostras independentes para comparar o desempenho das crianças do grupo disléxico e do grupo controle. $\mathrm{O}$ valor de alfa foi estabelecido em 0,05.

\section{Resultados}

A TABELA 1 mostra os valores médios e os respectivos desvios padrão das variáveis $\mathrm{FP}_{\text {Max }} \mathrm{e}$ tempo de execuçáo do teste dos 9-PnB, para o grupo controle e para o grupo disléxico. O teste $t$ de student não revelou diferença entre grupos para a $\mathrm{FP}_{\text {Max }}[\mathrm{t}(58)=0,5, \mathrm{p}>0,05$, bicaudal, Cohen's $\mathrm{d}=0,13]$. Porém, com relação ao tempo de execução do teste dos 9-PnB, teste $t$ student revelou que as crianças do grupo controle realizaram o teste em menor tempo do que as crianças disléxicas $[\mathrm{t}(58)=2,63, \mathrm{p}<0,01$, unicaudal, Cohen's $\mathrm{d}=0,69]$.

TABELA 1 - Valores médios (DP) das variáveis força de preensão máxima $\left(\mathrm{FP}_{\text {Max }}\right)$ e tempo gasto para execução do teste dos nove pinos nos buracos (9-PnB) realizados com a mão dominante por crianças do grupo controle e crianças com dislexia

\begin{tabular}{lcc}
\hline Grupos & FP $_{\text {Max }}(\mathbf{k g f})$ & Tempo 9-PnB (s) \\
\hline Dislexia & $19,66(6,66)$ & $17,65(1,93)^{\mathrm{a}}$ \\
Controle & $20,51(6,26)$ & $16,43(1,65)^{\mathrm{b}}$ \\
\hline
\end{tabular}




\section{Discussão}

Os resultados do presente estudo indicaram que crianças com dislexia apresentam desempenho inferior no teste dos 9-PnB quando comparadas com crianças sem dislexia. Diferentemente, não foi observada diferença entre crianças com e sem dislexia na produção de força de preensão palmar máxima. Os resultados do presente estudo indicam que crianças com dislexia apresentam alteraçóes na destreza dos dedos. Diferentemente, crianças com dislexia não apresentam alteraçóes na capacidade de geração de força, que representa a ação do sistema motor sem influência da captaçáo e processamento de informação sensorial.

A observação de que crianças com dislexia necessitam de um tempo maior para completar o teste dos 9-PnB indica que essas crianças apresentam algum comprometimento em tarefas que necessitam destreza dos dedos, além das dificuldades relacionadas com a escrita. Os resultados do presente estudo corroboram os resultados obtidos por LesLie, DAVIDSON e BATEY ${ }^{19} \mathrm{e}$ FAWCETT e NiCOLSON (1995), que também observaram que crianças com dislexia apresentaram desempenho inferior às crianças da mesma faixa etária em testes que exigem destreza dos dedos. Leslie, Davidson e BATEY $^{19}$ utilizaram o teste Perdue Pegboard para avaliar a destreza dos dedos de 23 crianças com e 23 crianças sem dislexia. O teste Perdue Pegboard é realizado com a mão dominante, com a não dominanante e com ambas as mãos sendo usadas simultaneamente (bimanual). Neste teste o participante deve colocar pinos pequenos em buracos pequenos que são apresentados num tabuleiro. No teste unimanual se pega o pino com a mão testada, que leva o mesmo para ser encaixado no buraco localizado no mesmo lado do tabuleiro e no teste bimanual a mão direita e mão esquerda pegam pinos simultaneamente e os colocam nos buracos, os da mão direita nos buracos da direita e os da esquerda nos buracos da mão esquerda. $\mathrm{O}$ escore do teste é o número de pinos colocados em 30 segundos.

LesLIE, DAvidson e BATEY ${ }^{19}$ observaram, ainda, que crianças com dislexia tiveram desempenho inferior ao das crianças sem dislexia nos testes realizados com a mão dominante e com a não dominante. Porém, não foi observada diferença entre grupos na tarefa bimanual. Os autores associaram esse pior desempenho de crianças disléxicas, observado quando o teste foi realizado pelo membro superior direito, a disfunções no hemisfério esquerdo, principalmente da área motora responsável pelo controle de tarefas motoras finas e a de açóes sequenciais. No estudo de FawCETT e NiCOLSON ${ }^{1}$, crianças com dislexia de 8 e de 13 anos e adolecentes de 17 anos realizaram o teste pegboard de forma mais lenta que crianças e adolecentes sem dislexia da mesma idade. Esses autores interpretaram o desempenho inferior de crianças e adolescentes com dislexia como um sinal de déficit sensório-motor, que tal déficit persiste até próximo da idade adulta e, ainda, que tal déficit pode estar associado à possíveis alteraçóes estruturais no cerebelo, haja vista que o cerebelo é a região onde esse processamento sensório-motor ocorre.

Embora seja importante saber a causa ou causas da dislexia, a criação e uso de um teste para o diagnóstico ou indicativo precoce da dislexia também é uma necessidade premente e, consequentemente, objetivo que precisa ser almejado. A identificaçáo de um teste que possibilite o diagnóstico precoce certamente auxiliaria educadores e terapeutas na aplicação de intervençóes que minimizem os efeitos indesejáveis e deletérios da dislexia durante a idade escolar e, em muitos casos, ao longo da vida. Com base nos resultados obtidos, podemos sugerir que um dos candidatos seria o teste dos 9-PnB, pois observamos que ele foi sensível para detectar diferença entre crianças com e sem dislexia. Além de sensível, o teste dos 9-PnB apresenta diversas outras características positivas, sendo um teste simples, de aplicação rápida (menos de 3 minutos) e de fácil entendimento pelo indivíduo avaliado. Mais ainda, esse teste poderia ser aplicado em crianças em idade pré-escolar ( 4 anos em diante $)^{28}$ e o resultado obtido por cada criança poderia ser comparado com seus pares ou com dados normativos para que essas crianças fossem classificadas como tendo ou náo risco de problema sensório-motor. O diagnóstico da dislexia é iniciado apenas após a criança ter iniciado as atividades de alfabetizaçáo, porém, se pudermos fazer uso de um teste sensório-motor simples poderemos identificar crianças com risco de desenvolver dislexia antes que os problemas de linguagem apareçam.

Ainda não existem dados normativos para a população brasileira e especificamente, para as crianças brasileiras. Porém, esforços têm sido dispendidos pelo nosso grupo, com a realização de estudo com uma amostra de crianças representativas para a obtenção de valores normativos e curvas de desenvolvimento do desempenho do teste dos 9-PnB. Com isso, poderemos identificar as crianças com risco de desenvolver alteraçóes sensório-motoras e usar esse teste como meio de diagnóstico auxiliar de desordens desenvolvimentais.

O presente estudo também examinou a capacidade de produção de força de preensão palmar máxima 
e mostrou que crianças com dislexia são capazes de gerar quantidade de força máxima similar às crianças sem dislexia. Além de propiciar conhecimento inédito, pois nenhum outro estudo foi encontrado com tal propósito, o resultado obtido corrobora a ideia que o problema das crianças com dislexia está no processamento sensório-motor, especificamente, na capacidade de utilizar as informações sensoriais disponíveis para a realização de açôes motoras de forma eficiente ${ }^{14,24,30}$. Crianças com dislexia não apresentam qualquer diferença em comparação com crianças não disléxicas em produzir força quando o uso de informação sensorial não tem um papel crucial para alcançar tal propósito. Com base nos resultados observados no presente estudo, pode-se sugerir que crianças com dislexia apresentam essa capacidade preservada e, portanto, testes de força máxima não têm qualquer utilidade na avaliação dessas crianças e até mesmo testes que não envolvam requisitos sensórios-motores.

Em resumo, podemos concluir que crianças com dislexia apresentam pior desempenho em uma tarefa manipulativa que exige destreza dos dedos quando comparadas às crianças sem diagnóstico de dislexia, o que indica que estas crianças apresentam déficits sensório-motores. Todavia, crianças com dislexia e sem dislexia produzem força de preensão palmar máxima em quantidade similar, o que mostra que crianças com dislexia apresentam o sistema motor preservado. Assim, esses resultados indicam que o problema da criança com dislexia não é no sistema motor e sim no modo como ela integra as informaçóes sensoriais e as respostas motoras para produzir a ação. Finalmente, o teste dos 9-PnB pode constituir ferramenta auxiliar importante para a identificação de crianças com dislexia, mesmo em idades tenras.

\section{Abstract}

Assessment of digits dexterity and power grip strength in dyslexic children

Dyslexia is characterized by difficulties in reading, writing and spelling, with no apparent cause. Children and adults with dyslexia also have deficits in different sensory-motor tasks. However, there is no consensus about the effect of dyslexia on digits dexterity and if there are purely motor changes in individuals affected by this disorder. The aim of the study was to compare children with and without dyslexia on digits dexterity and on power grip strength. Thirty children with dyslexia and 30 without dyslexia, between 8 and 14 years, performed the nine hole peg test (9HPT) for the evaluation of digits dexterity and maximum power grip strength (PGS) test, both with the dominant hand. They were instructed to perform the 9HPT as quickly as possible and then produce maximum PGS in the hydraulic dynamometer Jamar ${ }^{\mathrm{T} m}$. The shortest time and highest PGS recorded in three trials were used for statistical analysis. The results revealed that children with dyslexia are slower in performing 9HPT, but have the same ability to produce PGS than non-dyslexic children. These results indicate that deficits in digits dexterity experienced by dyslexic children do not originate in the motor system but in the way the children with dyslexia process sensory information and turns them into responses to produce motor actions.

KeYwords: Hand Function; Coordination; Manipulative Skills; Motor Control.

\section{Conflito de interesse}

Não há qualquer conflito de interesse.

\section{Referências}

1. Fawcett AJ, Nicolson RI. Persistent Deficits in Motor Skill of Children with Dyslexia. J Mot Behav. 1995;27(3):235-41.

2. Helmuth L. Neuroscience. Dyslexia: same brains, different languages. Science. 2001;16;291(5511):2064-5. 
3. Levelt WJ. Defining dyslexia. Science. 2001;18;292(5520):1300-1.

4. Paulesu E, Demonet JF, Fazio F, et al. Dyslexia: cultural diversity and biological unity. Science. 2001;16;291(5511):2165-7.

5. Ramus F. Dyslexia. Talk of two theories. Nature. 2001; 26;412(6845):393-5.

6. Katzir T, Kim YS, Wolf M, Morris R, Lovett MW. The varieties of pathways to dysfluent reading: comparing subtypes of children with dyslexia at letter, word, and connected text levels of reading. J Learn Disabil. 2008;41(1):47-66.

7. Paulesu E, Danelli L, Berlingeri M. Reading the dyslexic brain: multiple dysfunctional routes revealed by a new meta-analysis of PET and fMRI activation studies. Front Hum Neurosci. 2014;8:830.

8. Ramus F, Rosen S, Dakin SC, et al. Theories of developmental dyslexia: insights from a multiple case study of dyslexic adults. Brain. 2003;126(4):841-65.

9. Facoetti A, Paganoni P, Turatto M, Marzola V, Mascetti GG. Visual-spatial attention in developmental dyslexia. Cortex. 2000;36(1):109-23.

10. Skottun BC. Is dyslexia caused by a visual deficit? Vision Res. 2001;41(23):3069-71.

11. Stein J. The magnocellular theory of developmental dyslexia. Dyslexia. 2001;7(1):12-36.

12. Stein J, Talcott JB. Impaired neuronal timing in developmental dyslexia: the Magnocellular Hypothesis. Dyslexia. 1999;5(1):59-77.

13. Freitas PB, Pedão ST, Barela JA. Visuomotor processing and hand force coordination in dyslexic children during a visually guided manipulation task. Res Dev Disabil. 2014;35(10):2352-8.

14. Barela JA, Dias JL, Godoi D, Viana AR, Freitas PB. Postural control and automaticity in dyslexic children: the relationship between visual information and body sway. Res Dev Disabil. 2011;32(5):1814-21.

15. Fawcett AJ, Nicolson RI. Dyslexia: the role of the cerebellum. Electr J Res Educ Psychol. 2004;2(2):35-58.

16. Nicolson RI, Fawcett AJ, Dean P. Dyslexia, development and the cerebellum. Trends Neurosci. 2001;24(9):515-6.

17. Nicolson RI, Fawcett AJ, Dean P. Developmental dyslexia: the cerebellar deficit hypothesis. Trends Neurosci. 2001;24(9):508-11.

18. Rae C, Harasty JA, Dzendrowskyj TE, et al. Cerebellar morphology in developmental dyslexia. Neuropsychologia. 2002;40(8):1285-92.

19. Leslie SC, Davidson RJ, Batey OB. Purdue pegboard performance of disabled and normal readers: unimanual versus bimanual differences. Brain Lang. 1985;24(2):359-69.

20. Galaburda A, Livingstone M. Evidence for a magnocellular defect in developmental dyslexia. Ann N Y Acad Sci. 1993;14;682:70-82.

21. Stein J, Walsh V. To see but not to read; the magnocellular theory of dyslexia. Trends Neurosci. 1997;20(4):147-52.

22. Brookes RL, Tinkler S, Nicolson RI, Fawcett AJ. Striking the right balance: motor difficulties in children and adults with dyslexia. Dyslexia. 2010;16(4):358-73.

23. Getchell N, Pabreja P, Neeld K, Carrio V. Comparing children with and without dyslexia on the Movement Assessment Battery for Children and the Test of Gross Motor Development. Percept Mot Skills. 2007;105(1):207-14.

24. Viana AR, Razuk M, Freitas PB, Barela JA. Sensorimotor integration in dyslexic children under different sensory stimulations. PloS One. 2013;8(8):e72719.

25. Oldfield RC. The assessment and analysis of handedness: the Edinburgh inventory. Neuropsychologia. 1971;9(1):97-113.

26. Lima KCA, Francisco MM, Freitas PB. Relação entre os desempenhos em diferentes testes frequentemente utilizados na avaliação da função manual. Fisioter Mov. 2012;25(3):517-24.

27. Mathiowetz V, Weber K, Kashman N, Volland G. Adult norms for the nine hole peg test of finger dexterity. Occup Ther J Res. 1985;5(1):24-38.

28. Poole JL, Burtner PA, Torres TA, et al. Measuring dexterity in children using the Nine-hole Peg Test. J Hand Ther. 2005;18(3):348-51.

29. Mathiowetz V, Kashman N, Volland G, Weber K, Dowe M, Rogers S. Grip and pinch strength: normative data for adults. Arch Phys Med Rehabil. 1985;66(2):69-74.

30. Razuk M, Barela JA. Dyslexic children suffer from less informative visual cues to control posture. Res Dev Disabil. 2014;35(9):1988-94.

\begin{tabular}{r|l} 
ENDEREÇO & \\
Paulo Barbosa de Freitas & Recebido para publicação: 16/10/2015 \\
Rua Galvão Bueno, 868 - Liberdade & Revisão: 10/12/2017 \\
01506-00o - São Paulo - SP - BRASIL & Aceito: 02/03/2018 \\
e-mail: defreitaspb@gmail.com &
\end{tabular}

